\section{Economic impact of personality disorders in UK primary care attenders}

\author{
ALISON RENDU, PAUL MORAN, ANITA PATEL, MARTIN KNAPP \\ and ANTHONY MANN
}

\author{
Background The economic impact of \\ personality disorders on UK health \\ services is unknown.
}

\begin{abstract}
Aims To test the hypothesis that people with personality disorders have higher mean health and non-health costs compared with those without personality disorders.
\end{abstract}

\begin{abstract}
Method Prospective cohort study design. A total of 303 general practice attenders were followed-up I year after they had been assessed for the presence of personality disorders. Costs were estimated in $€$ sterling at 1999 price levels.
\end{abstract}

Results The mean total cost for patients with personality disorders was $£ 3094$ (s. d. =5324) compared with $€ 1633$ (s. d. =3779) for those without personality disorders. Personality disorders were not independently associated with increased costs. Multivariate analyses identified the presence of a significant interaction between personality disorders and common mental disorders and increased total costs (coefficient $=499,95 \% \mathrm{Cl}$ |80. $\mid-626.2, P=0.002)$.

\section{Conclusions Personality disorders are not independently associated with increased costs. An interaction between personality disorders and common mental disorders significantly predicts increased total costs.}

\section{Declaration of interest This study} was supported by grants from the Medical Research Council and the Department of Health.
Patients with personality disorders have been reported to use psychiatric services excessively (Seivewright et al, 1991; Saarento et al, 1997). Nevertheless, the economic impact of personality disorders on the health service remains largely unexplored. Only one previously published study has examined the cost of this patient group. Smith et al (1995) estimated that the cost of personality disorders to the National Health Service (NHS) in 1986, was $£ 61.3$ million. However, this study relied on national data sources to measure prevalence and service costs and provided no comparative group. Using a sample of consecutive attenders at four general practices in the London area, we set out to test the hypothesis that people with a personality disorder have higher mean health and non-health costs compared with those without a personality disorder.

\section{METHOD}

\section{Recruitment and baseline assessments}

The study was a follow-up of a cohort of 303 primary care attenders who had been recruited and assessed 1 year previously for the presence of personality disorders. Full details of the method and the characteristics of the study have been published elsewhere (Moran et al, 2000). In brief, 374 patients were recruited from a convenience sample of four general practices in the London area. Personality was assessed using a revised ICD-10 and DSM-IV version of the Standardised Assessment of Personality (SAP; Pilgrim et al, 1993). The SAP is a semi-structured interview designed for use with an informant and generates data that can be used to make a diagnosis of personality disorder according to ICD-10 and DSM-IV criteria. The personalities of 303 participants were rated using the SAP. A total of $72(24 \%)$ of the patients were assessed as having at least one personality disorder according to ICD-10 and DSMIV criteria. Demographic information was also recorded at baseline.

\section{One year follow-up}

One year after recruitment all participants were sent a questionnaire by post, containing the 12-item General Health Questionnaire (GHQ-12; Goldberg, 1972), the physical function sub-scale of the SF-36 (Jenkinson et al, 1993), a life events questionnaire (Brugha \& Cragg, 1990) and a use of services questionnaire. The GHQ-12 is a 12-item self-report questionnaire that screens for the presence of psychiatric morbidity and specifically 'common mental disorders', such as anxiety and depression (an increase in GHQ-12 score indicates greater psychiatric morbidity). The physical function sub-scale of the SF-36 is a self-report measure of physical functioning (an increase in physical function score indicates better physical functioning).

The use of services questionnaire was a variant of the Client Service Receipt Inventory (CSRI; Beecham \& Knapp, 1992). This questionnaire was used to collect retrospective data on service utilisation in the last 6 months and covers the following domains: general practitioner (GP) consultations; practice nurse visits; hospital in-patient stays; hospital out-patient episodes; seeing a social worker; counselling and therapy contacts. Participants were asked about the duration of consultations and any costs incurred for private treatment. Information was also collected on employment status and time taken off work because of ill health.

Estimated unit costs were calculated for all services used, drugs prescribed and time taken off work because of illness. All costs were calculated in $£$ sterling at 1998/99 price levels. Service cost estimates were based on figures published in Netten et al (1999) and allowed for capital, overheads, travelling time, non-patient contact time, support services and London weighting. Where costs were not available in Netten et al, other sources and equivalent costs were used. Lost productivity costs, because of illness or unemployment, were calculated as the only non-health service costs. Figures were based on national average gross earnings by occupational status. The researcher responsible for 
costing the data (A.R.) was blind to the personality status of the participants.

\section{Sample size}

A pre-study power calculation showed that a sample of 300 participants would be required to detect a 50 unit difference in mean costs between participants with personality disorders and those without, with $80 \%$ power and $95 \%$ significance. This assumes a $30 \%$ prevalence of personality disorder (Casey \& Tyrer, 1990) and a standard deviation of 125 .

\section{Statistical analysis}

Analyses were performed using SPSS and Stata (Norusis, 1993; StataCorp, 1999). A strategy for the statistical analysis of the association between baseline measures and 1-year cost was drawn up before inspecting the data. The main outcome measures used were the arithmetic means for health service, non-health service and total costs. Univariate associations between personality disorder and costs were investigated using $t$-tests; the results were checked using non-parametric bootstrap analyses (based on 2000 replications). Multiple linear regression was then used to identify variables that predicted variations in costs. The following variables are associated with health service use and were therefore entered into the multiple regression models: age; gender; physical health and psychiatric morbidity (Gill \& Sharpe 1999; Dowrick et al, 2000). The following additional variables were entered into the multiple regression models on a priori grounds: personality disorder status, life events, marital status and age on finishing education (a proxy measure for socio-economic status). Interactions between personality disorder and SF-36 physical function score and General Health Questionnaire score were also included in the model.

Results from the multiple regression models were subject to two checks. First, they were compared with the results from non-parametric bootstrap regression to assess the robustness of confidence intervals and $P$ values to non-normality in the cost distribution (Efron \& Tibshirani, 1993). Confidence limits were obtained from bias-corrected estimates based on 2000 resamples; significance levels were obtained from percentiles of the distribution of bootstrapped re-samples. Second, the results were compared with those obtained from a generalised linear model, where a non-normal (gamma distribution) was assumed for costs.

\section{RESULTS}

\section{Completeness of follow-up}

Of the 303 participants rated at baseline, $254(84 \%)$ returned completed questionnaires. Participants with personality disorders were not over-represented among those lost to follow-up $\left(\chi^{2}=0.56\right.$; $P=0.81)$. Those lost to follow-up were more likely to be male $\left(\chi^{2}=3.97 ; P=0.05\right)$ and younger $(t$-test, 2 -tailed $P=0.01)$.

\section{Univariate analyses}

Mean costs were consistently higher for patients with personality disorders compared with those without personality disorders, although the difference was only statistically significant for total costs (Table 1). Non-parametric bootstrap analyses confirmed these findings.

Table I Mean costs, by personality disorder status

\begin{tabular}{lccc}
\hline Cost & $\begin{array}{c}\text { Personality disorder } \\
(n=60)\end{array}$ & $\begin{array}{c}\text { Non-personality disorder } \\
(n=192)\end{array}$ & $\begin{array}{c}t \text {-test } \\
P \text { value }\end{array}$ \\
\hline Total health service costs & $507.0(884.5)$ & $313.6(854.3)$ & 0.13 \\
Total non-health service costs & $2544.8(4994.8)$ & $1312.5(3584.7)$ & 0.08 \\
Total cost & $3094.3(5324.2)$ & $1632.9(3779.0)$ & 0.05 \\
\hline
\end{tabular}

Numbers appearing in the columns are mean costs $(£)$. Standard deviations are given in parentheses.

Table 2 Multivariate predictors of I-year health service costs $(£)$

\begin{tabular}{lcc}
\hline Multivariate predictor & Coefficient (95\% Cl)' & $P$ \\
\hline Age (years) & $-0.43(-9.8$ to 8.9$)$ & 0.92 \\
Gender (male compared with female) & $193(-33.9$ to 419.1$)$ & 0.10 \\
Marital status (married compared with being single) & $21(-196.7$ to 237.8$)$ & 0.85 \\
Age on finishing education (years) & $-54(-124.8$ to 16.4$)$ & 0.13 \\
Total number of life events & $55(-35.7$ to 145.6$)$ & 0.23 \\
PF score & $-46(-71.2$ to -20.0$)$ & $<0.001$ \\
GHQ-12 score & $32(-5.9$ to 69.8$)$ & 0.10 \\
Personality disorder status (non-personality disorder compared & $-719(-1994.2$ to 555.4$)$ & 0.27 \\
$\quad$ & & \\
with personality disorder) & $33(-12.8$ to 79.3$)$ & 0.16 \\
Interaction term between personality disorder and PF score & $-12(-83.4$ to 59.5$)$ & 0.74 \\
Constant & $1620(580.4$ to 2658.7$)$ & 0.003 \\
\hline
\end{tabular}

PF, SF-36 physical function sub-scale score.

I. For continuous variables (e.g. age) the coefficient indicates the increase or decrease in cost per unit increase in the covariate (e.g. per year of age). For categorical variables the coefficient is the difference in cost between the specified group and the comparison group indicated in brackets next to the variable name. Adjusted $R^{2}=0.12$.

\section{Multivariate analyses}

Health service costs

A percentage $(12 \%)$ of the variance of health service costs was explained by variables in the multivariate mode was significanty associated with healt was significantly associated with health costs; a one-point increase in function score was associated with associated with health service costs in this model. Bootstrap regression analysis and generalised linear modelling confirmed these findings.

\section{Non-health service costs}

A percentage (24\%) of the variance of nonhealth service costs was explained by variables in the multivariate model cost status. In addition, non-health service costs Personality disorder was not significantly 
were significantly associated with an interaction between personality disorder and GHQ-12 score. For those participants without a personality disorder, a one-point increase in GHQ-12 score was associated with a $£ 253$ increase in non-health service costs. For patients with personality disorders, a one-point increase in GHQ12 score was associated with a $£ 764$ increase in non-health service costs.

Bootstrap regression analyses largely confirmed these results, although marital status failed to remain significantly associated with non-health service costs. A generalised linear model of non-health service costs did not find the interaction between GHQ-12 score and personality disorder to be statistically significant.

\section{Total costs}

Some of the variance of total costs $(28 \%)$ was explained by variables in the multivariate model. Four variables were significantly associated with total costs:

Table 3 Multivariate predictors of I-year non-health service costs $(£)$

\begin{tabular}{|c|c|c|}
\hline Multivariate predictor & Coefficient $(95 \% \mathrm{Cl})^{\prime}$ & $P$ \\
\hline Age (years) & $I(-38 . I$ to 40.1$)$ & 0.96 \\
\hline Gender (male compared with female) & $|25|$ (305.4 to 2197.4$)$ & 0.01 \\
\hline Marital status (married compared with being single) & $-975(-1882.6$ to -67.4$)$ & 0.04 \\
\hline Age on finishing education (years) & $-279(-573.8$ to 16.0$)$ & 0.06 \\
\hline Total number of life events & $218(-160.1$ to 596.9$)$ & 0.26 \\
\hline PF score & $-54(-161.3$ to 52.4$)$ & 0.32 \\
\hline GHQ-12 score & 253 (95.3 to 414.2$)$ & 0.002 \\
\hline $\begin{array}{l}\text { Personality disorder status (non-personality disorder compared } \\
\text { with personality disorder) }\end{array}$ & $-1535(-6890.8$ to 3790.7$)$ & 0.57 \\
\hline $\begin{array}{l}\text { Interaction term between personality disorder and GHQ-12 } \\
\text { score }\end{array}$ & 511 (2I2.2 to 809.2$)$ & $<0.001$ \\
\hline Interaction term between personality disorder and PF score & $24(-168.2$ to 216.5$)$ & 0.81 \\
\hline Constant & $3602(-738.9$ to 7942.9$)$ & 0.1 \\
\hline
\end{tabular}

PF, SF-36 physical function sub-scale score.

I. For continuous variables (e.g. age) the coefficient indicates the increase or decrease in cost per unit increase in the covariate (e.g. per year of age). For categorical variables the coefficient is the difference in cost between the specified group and the comparison group indicated in brackets next to the variable name.

Adjusted $R^{2}=0.24$

Table 4 Multivariate predictors of I-year total costs $(t)$

\begin{tabular}{lcc}
\hline Multivariate predictor & Coefficient (95\% Cl)' & $P$ \\
\hline Age (years) & $0.59(-40.1$ to 41.3$)$ & 0.98 \\
Gender (male compared with female) & $1444(499.3$ to 2332$)$ & 0.004 \\
Marital status (married compared with being single) & $-955(-1923$ to -99.3$)$ & 0.05 \\
Age on finishing education (years) & $-333(-593.2$ to -38.5$)$ & 0.03 \\
Total number of life events & $273(-120.3$ to 667$)$ & 0.17 \\
PF score & $-100(-186$ to -4.2$)$ & 0.08 \\
GHQ-I2 score & $285(165$ to 474.7$)$ & 0.001 \\
Personality disorder status (non-personality disorder compared & $-2255(-7793.3$ to 3283.7$)$ & 0.42 \\
$\quad$ with personality disorder) & & 0.002 \\
Interaction term between personality disorder and GHQ-12 score & $499(180.1$ to 626.2) & 0.57 \\
Interaction term between personality disorder and PF score & $57(-142.7$ to 257.4$)$ & 0.02 \\
Constant & 5222 (683.7 to 9759.5$)$ & \\
\hline
\end{tabular}

PF, SF-36 physical function sub-scale score.

I. For continuous variables (e.g. age) the coefficient indicates the increase or decrease in cost per unit increase in the covariate (e.g. per year of age). For categorical variables the coefficient is the difference in cost between the specified group and the comparison group indicated in brackets next to the variable name.

Adjusted $R^{2}=0.28$

GHQ-12 score, gender, age on finishing education and marital status (Table 4).

In addition, an interaction between GHQ-12 score and personality disorder was significantly associated with total costs. For participants without personality disorders, a one-point increase in GHQ12 score was associated with a $£ 285$ increase in total costs. For participants with personality disorders, a one-point increase in GHQ-12 score was associated with a $£ 784$ increase in total service costs.

The non-parametric bootstrap analysis confirmed the presence of a statistically significant interaction between GHQ-12 score and personality disorder in association with total costs. However, a generalised linear model of total costs failed to confirm the presence of this interaction.

\section{DISCUSSION}

\section{Personality disorder and cost}

Personality disorder was not significantly associated with mean health or non-health service costs at either a univariate or multivariate level. Personality disorder was significantly associated with higher mean total costs, although this association failed to remain statistically significant in the multivariate analysis. Our main hypothesis was therefore not supported.

A significant interaction emerged between personality disorder and common mental disorders in the multivariate analyses of non-health and total costs; the costs for patients with personality disorder were significantly higher in the presence of common mental disorders. The presence of this interaction was confirmed by nonparametric bootstrap analyses (which assume an additive model of costs), although it was not confirmed by generalised linear modelling (which assumes a multiplicative model of costs). This makes us more cautious in attributing significance to the interaction. Nevertheless, it also raises methodological questions as to the need for multiple confirmatory analysis (Knapp et al, 2002).

\section{Evaluation of the study design}

This is the first published study of the economic impact of personality disorder in the UK health service. The study is characterised by three positive features. First, it is a prospective study in which the assessment of costs was not influenced by the assessment of personality. Second, the 
researcher costing the data was blind to the personality status of participants, therefore minimising observer bias in the estimation of costs. Third, the use of an informantbased assessment of personality minimised the possibility of concurrent abnormal mental state biasing the assessment of personality. Despite these features, the study has a number of limitations. First, the patients in the study were primary care attenders and are likely to be different from the patients seen by general psychiatrists. This therefore limits the extent to which the findings can be generalised to psychiatric populations. Second, a convenience and not a random sample of practices was used and the findings might not therefore be generalisable to patients from other UK practices. Third, our sample of consecutive attenders could have included a greater number of heavy service users. This could have inflated the costs for the whole sample, although it should not have affected the relative costs of attenders with personality disorders compared with attenders without personality disorders. Fourth, our power calculation was based on a prevalence of $30 \%$ for personality disorder, although the detected prevalence was only $24 \%$. Therefore, our study could have been underpowered to detect an association between personality disorder and cost. Underpowering is a problem common to health economic studies, especially in the mental health field (Gray et al, 1997), although in our case, we had used the best available prior data to try to avoid this difficulty. Finally, the basis for our measure of cost, the CSRI, is a self-report instrument and the estimated costs could, therefore, be susceptible to recall bias.

\section{Explained variation in cost}

Health service costs were associated with physical function status. This is consistent with the finding that heavy general practice service users are less likely to report excellent and good health and more likely to have physical disease, compared to controls (Gill \& Sharpe, 1999). However, our final regression model only explained $12 \%$ of the variance in total health service costs. Unmeasured variables of relevance to the prediction of health service costs could include factors such as cigarette and alcohol consumption, physical activity, access to services and socio-economic status (Knapp, 1998). Our finding of no association between personality disorder and

\section{CLINICAL IMPLICATIONS}

General practitioner (GP) patients with personality disorders have higher mean total costs compared with those without personality disorders.

- Physical function was significantly associated with health service costs. Common mental disorders were significantly associated with both non-health service costs and total costs.

- An interaction between personality disorders and common mental disorders was significantly associated with non-health service costs and total costs. These increased costs could be incurred as a result of the chronicity of associated mental illness.

\section{LIMITATIONS}

The results apply to a population of GP attenders and might not be generalisable to populations of patients with psychiatric disorders.

- The multiple regression models explained only a small proportion of the variations in cost.

- The study could have failed to identify and measure variables that have a significant impact on cost.

ALISON RENDU, BA, Centre for the Economics of Mental Health; PAUL MORAN, MRCPsych, Health Services Research Department; ANITA PATEL, MSc, Centre for the Economics of Mental Health, Institute of Psychiatry, London; MARTIN KNAPP, PhD, Centre for the Economics of Mental Health, Institute of Psychiatry; and PSSRU, London School of Economics, UK; ANTHONY MANN, FRCPsych, Section of Epidemiology, Institute of Psychiatry, London

Correspondence: Paul Moran, Health Services Research Department, Institute of Psychiatry, De Crespigny Park, London SE5 8AF, UK. Tel: 0207848 0568; fax: 0207848 0333; e-mail:

paul.moran@iop.kcl.ac.uk

(First received 26 September 200I, final revision 14 March 2002, accepted 2I March 2002)

health service costs is at odds with previous reports of an association between personality disorder and high psychiatric service utilisation (Seivewright $e t$ al, 1991; Saarento et al, 1997). However, as noted above, this could reflect the fact that, despite our best efforts, the study was underpowered to detect such an association.

In our sample of GP attenders, the presence of common mental disorders predicted both non-health service costs (lost productivity) and total costs. This finding is consistent with other reports of a significant association between common mental disorders and disability (Ormel et al, 1993). However, our findings suggest that common mental disorders interacted with personality disorders in predicting both non-health service and total costs; patients with personality disorders were only more expensive if they also had common mental disorders. Personality disorders are often associated with a poor prognosis for the treatment of associated mental illness (Patience et al, 1995; Mennin \& Heimberg, 2000). We therefore suggest that increased total costs for the patients with personality disorders could have occurred as a result of chronicity of associated mental illness (Seivewright et al, 1998).

Personality disorders are currently the subject of great debate. However, the current debate is focused on the small proportion of individuals who have a 'severe personality disorder' and could pose a danger to the public. By examining the economic impact of the whole diagnostic group of patients with personality disorders, we have shown that personality disorders could have a subtle effect on non-health service and total costs through an interaction with psychiatric comorbidity. Clearly our findings need replication in a larger, more representative sample of patients. However, we believe that this 
study is an important step towards improving the evidence base in an area that is overburdened with opinion rather than fact.

\section{ACKNOWLEDGEMENTS}

We would like to thank Morven Leese and Jack Astin for their assistance with data analysis and Lisa Green and Saddaff Piracha for help in recruitment of participants and entering data. We are grateful to the patients, informants and GPs that participated in this study. Finally, we want to thank two anonymous referees for their comments on an earlier draft of this paper. The study was supported by grants from the Department of Health and the Medical Research Council.

\section{REFERENCES}

Beecham, J. \& Knapp, M. R. J. (1992) Costing psychiatric interventions. In Measuring Mental Health Needs (eds G. Thornicroft, C. R. Brewin \& J.Wing), pp. 163-183. London: Gaskell.

Brugha, T. \& Cragg, D. (1990) The list of threatening experiences: the reliability and validity of a brief life events questionnaire. Acta Psychiatrica Scandinavica, 82, $77-81$.

Casey, P. R. \& Tyrer, P. (1990) Personality disorder and psychiatric illness in general practice. British Journal of Psychiatry, 156, 261-265.

Dowrick, C., Bellon, J. \& Gomez, M. (2000) GP frequent attendance in Liverpool and Granada: the impact of depressive symptoms. British Journal of General practice, 50, 36I-365.
Efron, B. \& Tibshirani, R. J. (1993) An Introduction to the Bootstrap. New York: Chapman and Hall.

Gill, D. \& Sharpe, M. (1999) Frequent consulters in general practice: a systematic review of studies of prevalence, associations and outcome. Journal of Psychosomatic Research, 47, II5-130.

Goldberg, D. (1972) The Detection of Psychiatric Illness by Questionnaire. (Vol. 21). Oxford: Oxford University Press.

Gray, A. M., Marshall, M., Lockwood, A., et al (1997) Problems in conducting economic evaluations alongside clinical trials. Lessons from a study of case management for people with mental disorders. British Journal of

Psychiatry, I70, 47-52.

Jenkinson, C., Wright, L. \& Coulter, A. (1993) Quality of life measurement in health care. A review of measures and population norms for the UK SF-36. Oxford: Health Services Research Unit.

Knapp, M. (1998) Making music out of noise - the cost function approach to evaluation. British journal of Psychiatry, 173 (suppl. 36), 7-II.

_ , Chisholm, D., Leese, M., et al (2002) Comparing patterns and costs of schizophrenia care in five European countries: the EPSILON study. Acta Psychiatrica Scandinavica, 105, 42-54.

Mennin, D. S. \& Heimberg, R. G. (2000) The impact of comorbid mood and personality disorders in the cognitive-behavioral treatment of panic disorder. Clinical Psychology Review, 20, 339-357.

Moran, P., Jenkins, R., Tylee, A., et al (2000) The prevalence of personality disorder among primary care attenders. Acta Psychiatrica Scandinavica, 102, 52-57.

Netten, A., Dennett, J. \& Knight, J. (1999) Unit Costs of Health and Social Care. University of Kent, Canterbury: Personal Social Services Research Unit.
Norusis, M. (1993) SPSS for Windows. Base systems user's guide. Release 6.0. Chicago: SPSS

Ormel, J., Korff, M. V., Brink, W. v. D., et al (1993) Depression, anxiety and social disability show synchrony of change in primary care patients. American Journal of Public Health, 83, 385-390.

Patience, D. A., McGuire, R. J., Scott, A. I., et a (1995) The Edinburgh Primary Care Depression Study: personality disorder and outcome. British Journal of Psychiatry, 167, 324-330.

Pilgrim, J., Mellers, J., Boothby, H., et al (1993) Interrater and temporal reliability of the Standardised Assessment of Personality and the influence of informant characteristics. Psychological Medicine, 23. 779-786.

Saarento, O., Nieminten, P., Hakko, H., et al (1997) Utilization of psychiatric in-patient care among new patients in a comprehensive community-care system: 3 -year follow-up study. Acta Psychiatrica Scandinavica, 95, 132-139.

Seivewright, H., Tyrer, P., Casey, P., et al (1991) A three-year follow-up of psychiatric morbidity in urban and rural primary care. Psychological Medicine, 21 495-503.

_, _ \& Johnson, T. (1998) Prediction of outcome in neurotic disorder: a 5-year prospective study. Psychological Medicine, 28, II49-II57.

Smith, K., Shah, A., Wright, K., et al (1995) The prevalence and costs of psychiatric disorders and learning disabilities. British Journal of Psychiatry, 166, 9-18.

StataCorp (1999) Stata Statistical Software, Release 6.0. College Station, TX: Stata Corporation. 